
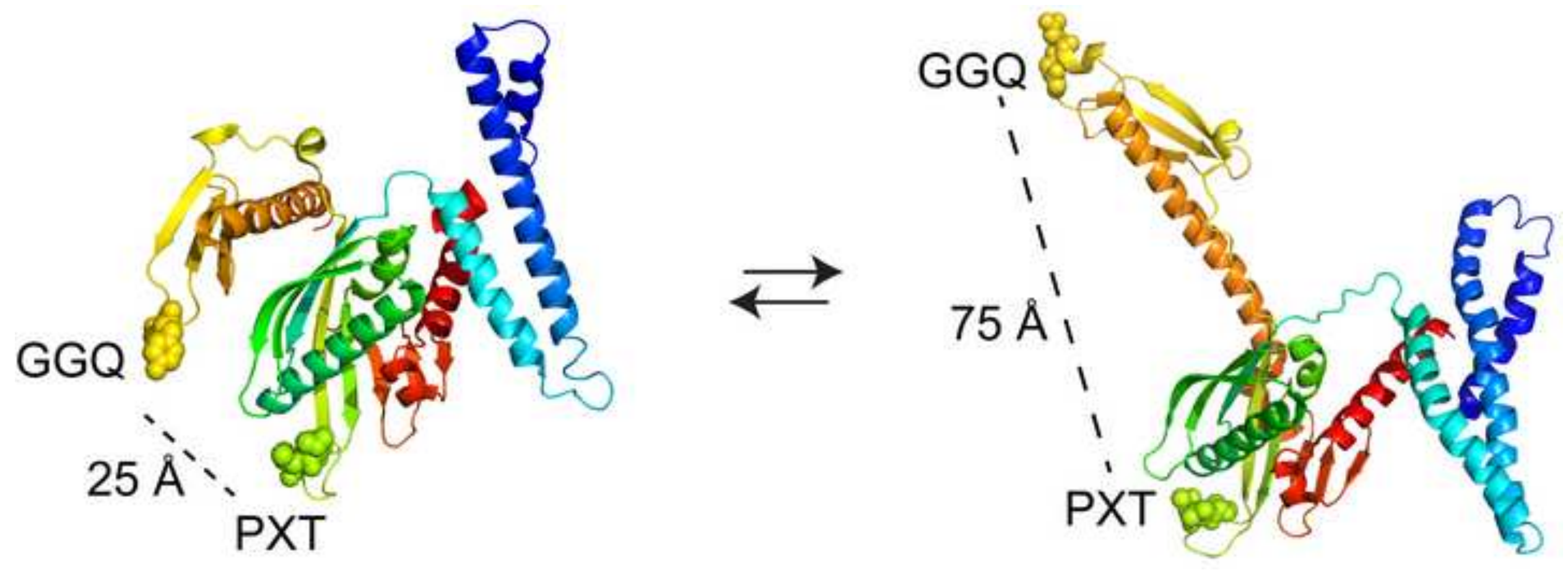


\title{
Ribosome Induces a Closed to Open Conformational Change in Release Factor 1
}

\author{
Krista Trappl and Simpson Joseph \\ Department of Chemistry and Biochemistry, University of California at San Diego, 9500 \\ Gilman Drive, La Jolla, California \\ 92093-0314, United States
}

Corresponding author:

Simpson Joseph

4102 Urey Hall,

Department of Chemistry and Biochemistry,

University of California at San Diego,

9500 Gilman Drive, La Jolla, CA 92093-0314.

Phone: (858) 822-2957

E-mail: sjoseph@ucsd.edu 


\begin{abstract}
Bacterial translation termination is triggered when a stop codon arrives at the ribosomal A site. Stop codons are recognized by class I release factors (RF1 and RF2 in E. coli), which bind to the ribosome and catalyze the release of the newly synthesized protein. Crystal structures showed that RF1 and RF2 are in an open conformation when bound to the ribosome but is in a closed conformation when not bound to the ribosome. It is not clear whether only the open form of RF1 and RF2 bind to the ribosome. Alternatively, the closed form of RF1 and RF2 may bind to the ribosome and undergo a conformational change to the open state upon binding. We used transition metal ion FRET experiments (tmFRET) to monitor precisely the conformation of RF1 in the absence and the presence of the ribosome. Our results indicate that RF1 undergoes a large conformational change from a closed to an open form upon binding to the ribosome. Our results are consistent with the mechanism, in which high termination fidelity is achieved by linking stop codon recognition by RF1 to the change in conformation from closed to open state.
\end{abstract}

\title{
Keywords:
}

translation termination; release factors; stop codons; peptide release; tmFRET

\begin{abstract}
Abbreviations used:
tmFRET, transition metal ion fluorescence resonance energy transfer; RF, release factor; $\mathrm{RC}$, release complex; SAXS, small-angle X-ray scattering;
\end{abstract}




\section{Introduction}

Bacterial class I release factors 1 and 2 (RF1 and RF2) are responsible for recognizing stop codons in the ribosomal A site and catalyze the release of newly synthesized proteins from the ribosome [1]. RF1 recognizes UAA and UAG, whereas RF2 recognizes UAA and UGA [2]. RF1 and RF2 contain four domains with domains II and IV forming a stable structural core to which domains III and I are flexibly connected [3-6]. Conserved residues in domain II of RF1 and RF2 recognize stop codons in the decoding center of the 30S ribosomal subunit [7-10]. A GGQ motif present in domain III of both factors is important for inducing peptide release by interacting with the peptidyl transferase center located in the 50S ribosomal subunit [7-10]. Thus, RF1 and RF2 in this "open" conformation span the distance of $75 \AA$ between the decoding and peptidyl transferase centers of the ribosome [11]. However, RF1 and RF2 have been crystalized in a "closed" conformation in the absence of the ribosome $[3,4,12]$. In this closed conformation, RF1 and RF2 cannot span the distance between the decoding and the peptidyl transferase centers. It is not clear whether RF1 and RF2 when free in solution exist in the open conformation or whether binding to the ribosome is essential for inducing the open conformation. In addition, it is not known whether the closed to open conformational change by RF1 and RF2 is coupled to stop codon recognition and peptide release.

To determine the conformation of RF1 and RF2 in solution, a small-angle X-ray scattering (SAXS) technique was used previously. One SAXS study showed that E. coli RF1 is in the open conformation even in the absence of the ribosome suggesting that the open structure of RF1 is stable in solution, and that binding to the ribosome is not 
essential for inducing the closed to open conformational change [13]. However, another SAXS study concluded that Thermus thermophilus RF2 is mostly in the closed conformation in solution [14]. As mentioned above, RF1 and RF2 crystal structures are in the closed conformation $[3,4,12]$. The crystal structure of E. coli RF1 in complex with the RF methyltransferase PrmC is also in the closed conformation [12]. Finally, SAXS provides information about the overall average shape of the protein ensemble, and sometimes multiple conformations of the protein may lead to ambiguity in the interpretation of the data. Therefore, the conformation of RF1 and RF2 when free in solution remains an open question.

To better understand the conformation of RF1 in the absence and presence of the ribosome, we made use of a newly developed variation of FRET called transition metal ion FRET (tmFRET) [15-18]. Classical FRET uses fluorophores with long linkers whose orientation factors $(\kappa)$ are difficult to determine and have large Förster critical distances $\left(R_{0}\right)$ making it difficult to monitor precisely intra-molecular rearrangements in smaller proteins [19]. In contrast, tmFRET uses a colored metal ion complex bound to a dihistidine motif as acceptor and a non-standard fluorophore with a shorter linker as a donor to monitor small-scale rearrangements in the range of 5 to $20 \AA$ [17]. Thus, tmFRET is ideal for monitoring the conformational change in RF1. By selecting suitable positions for the di-histidine motif and the fluorophore (bimane) in RF1, it will be possible to monitor the closed and open conformation of RF1. In the closed RF1 conformation, the distance between the coordinated metal ion and bimane is small resulting in quenching of the fluorescence of bimane (Fig. 1, left). Whereas, in the open RF1 conformation, the distance between the coordinated metal ion and bimane is larger 
resulting in decreased quenching of the fluorescence of bimane (Fig. 1, right). We used tmFRET to monitor the structure of RF1 in solution and when bound to ribosomes with either a stop or sense codon in the A site. Interestingly, our results show that RF1 free in solution is mainly in the closed conformation and changes to the open conformation in the presence of ribosomes with a stop codon in the A site. In the absence of a proofreading mechanism during termination, it is possible that the closed to open conformational change by RF1 and RF2 is used to enhance the accuracy of stop codon recognition.

\section{Materials and Methods:}

\section{Buffers, Ribosomes, tRNA, mRNA preparation, wild type RF1}

All experiments were carried out in buffer A $(6 \mathrm{mM}$ HEPES pH 7.5, $10 \mathrm{mM} \mathrm{MgCl}$, $150 \mathrm{mM} \mathrm{NH}_{4} \mathrm{Cl}_{2}$ ). Tight-coupled $70 \mathrm{~S}$ ribosomes were isolated from E. coli MRE600 cells as described earlier [20]. Native tRNA ${ }^{\mathrm{fMet}}$ was overexpressed in E. coli and purified by HPLC [21]. Synthetic mRNAs with either a UAA stop codon or a UUU sense codon after the AUG start codon were purchased from Dharmacon [22]. The sequence of the mRNA is 5'-AAG GAG GUA AAA AUG UAA GCU -3' (the stop codon is indicated in bold). Wild type RF1 was purified as described previously [22].

\section{Mutant RF1 preparation}

Mutant RF1 was produced by site-directed mutagenesis (QuickChange, Stratagene). Starting from a cysteine-free RF1 gene in pPROEx-HTc vector (Invitrogen), single 
cysteine mutations were introduced first, followed by the double-histidine mutations. RF1 mutant proteins were purified by nickel-affinity chromatography. The His-tag was removed by TEV protease, and RF1 was concentrated using a $10 \mathrm{kD}$ MWCO spin column (Amicon Ultra-15, EMD Millipore).

\section{Fluorophore Labeling of RF1 mutants}

A $1 \mathrm{ml}$ aliquot of RF1 mutants (100 $\mu \mathrm{M}$ final conc.) in labeling buffer (50 mM HEPES $\mathrm{pH} 7.5,300 \mathrm{mM} \mathrm{NaCl}$ ) was incubated with 10 -fold excess (1 mM final conc.) of monobromobimane (mBBr) (Sigma-Aldrich) in the dark for $2-4$ hours. The excess dye was removed by gel filtration (Bio-Spin 6 column, BioRad). Subsequently the protein was dialyzed against $2 \mathrm{~L}$ of protein storage buffer $(50 \mathrm{mM}$ Tris- $\mathrm{Cl} \mathrm{pH} 8.0,300 \mathrm{mM}$ $\mathrm{NaCl})$ in the dark overnight and stored in $20 \mu \mathrm{L}$ aliquots at $-80{ }^{\circ} \mathrm{C}$ after flash freezing with liquid nitrogen.

\section{RF1 binding assay with pyrene-labeled mRNA}

Release complexes (RC) were formed using pyrene-labeled mRNA having a UAA stop codon in the ribosomal A-site, as described before [22]. $\mathrm{RC}(0.25 \mu \mathrm{M}$ final conc.) were incubated at room temperature with wild type RF1 or bimane-labeled mutant RF1 ( $0.5 \mu \mathrm{M}$ final conc.) for 60 minutes. This experiment was performed in the absence of $\mathrm{Cu}(\mathrm{II})$. Samples were excited at $343 \mathrm{~nm}$ and fluorescence emission intensity was scanned from 360 to $420 \mathrm{~nm}$ with a fluorometer (Fluoromax-P, J.Y. Horiba Inc.). The emission peak at $376 \mathrm{~nm}$ was used for calculating the fraction of RF1 bound to the ribosome. 


\section{Peptide release assay}

Peptide release time courses with wild type RF1 and bimane-labeled mutant RF1 were performed in the absence of $\mathrm{Cu}(\mathrm{II})$ and in the presence of increasing concentrations of $\mathrm{Cu}(\mathrm{II})(0-100 \mu \mathrm{M})$, as described before [22]. The final concentration for RF1 and RC were $40 \mu \mathrm{M}$ and $0.5 \mu \mathrm{M}$, respectively. Samples were spotted on an eTLC plate, separated and analyzed as described previously [22]. All experiments were repeated at least three times. Values were normalized, and the peptide release rates were calculated using the single exponential equation $\mathrm{Y}=\mathrm{Ymax} *(1-\exp (-\mathrm{K} * \mathrm{X}))$.

\section{$\mathrm{Cu}($ II) titration experiment without $\mathrm{RC}$}

Bimane-labeled RF1 mutants $(0.3 \mu \mathrm{M}$ final conc.) were incubated with increasing amounts of $\mathrm{Cu}$ (II) (0 to $10 \mathrm{mM}$ final conc.) for 10 minutes at room temperature in the dark in black, non-binding 96-well microplates (Bio-One, Greiner) (200 $\mu$ l final reaction volume). The plate was transferred to a fluorescence microplate reader (Safire 2, Tecan) and each sample was excited at $400 \mathrm{~nm}$ (12 nm slits) and the fluorescence emission intensity at $460 \mathrm{~nm}$ (6 nm slits) was measured. For each well, 60 reads were taken with an integration time of 1 second. The experiments were done in triplicates and were repeated at least two times to calculate the standard deviations.

\section{$\mathrm{Cu}(\mathrm{II})$ titration experiment with $\mathrm{RC}$}

RC was formed with unlabeled mRNA having either a UAA stop codon or a UUU sense codon in the A site, as described above. Bimane-labeled RF1 mutants $(0.3 \mu \mathrm{M}$ final conc.) were pre-incubated for 10 minutes with increasing amounts of $\mathrm{Cu}(\mathrm{II})(0-1 \mathrm{mM}$ 
final conc.) followed by the addition of $\mathrm{RC}(0.3 \mu \mathrm{M}$ final conc.) and the incubation at room temperature in the dark was continued for 2 hours. Samples were excited at 400 $\mathrm{nm}$ (4 nm slits), and the fluorescence emission intensity at $460 \mathrm{~nm}$ (4 nm slits) was measured with a fluorometer (Fluoromax-P, J.Y. Horiba Inc.). The experiments were repeated at least three times to calculate the standard deviations.

\section{tmFRET with RC Titration}

For RC titration experiments a final concentrations of $0.1 \mu \mathrm{M}$ bimane-labeled mutant $\mathrm{RF} 1$ and $50 \mu \mathrm{M} \mathrm{Cu}(\mathrm{II}) \mathrm{Cl}_{2}$ were pre-incubated for 10 minutes at room temperature in the dark followed by the addition of increasing amounts of $\mathrm{RC}(0.01 \mu \mathrm{M}$ to $0.4 \mu \mathrm{M}$ final conc.) and the incubation was continued for 2 hours at room temperature in the dark. Fluorescence emission intensities of the samples were determined with a fluorometer, as described above.

\section{Fluorescence Data Analysis}

The fluorescence intensity data were fit to the following single-site binding equation:

$$
\frac{F_{\text {metal }}}{F}=\frac{1}{1+\frac{[\text { metal }]}{K d 1}}
$$

where, $F_{\text {metal }}$ is the fluorescence in the presence of $\mathrm{Cu}(\mathrm{II})$ and $F$ is the fluorescence in the absence of $\mathrm{Cu}(\mathrm{II}) . K_{\mathrm{d} 1}$ is the dissociation constant for free $\mathrm{Cu}(\mathrm{II})$ in solution, which leads to collisional quenching of the fluorophore. For RF1 mutants with the His clamp, the data were fit to the following two-site binding equation: 


\section{FRET efficiency and distance calculations from tmFRET}

The FRET efficiency (E) was calculated from

$$
E=1-\frac{F_{\text {Metal }}}{F}
$$

where, $F$ is the fluorescence signal of RF1 with $\mathrm{RC}$ in the absence of $\mathrm{Cu}(\mathrm{II})$ and $F_{\text {Metal }}$ is the fluorescence signal of RF1 with $\mathrm{RC}$ in the presence of $50 \mu \mathrm{M} \mathrm{Cu}(\mathrm{II})$. We also calculated FRET efficiency by using the fluorescence data of RF1 mutant with the His clamp and its corresponding cysteine-only control in the presence of $50 \mathrm{nM} \mathrm{Cu}$ (II) and obtained similar values for FRET efficiency with both methods.

The distance (R-value) between acceptor $[\mathrm{Cu}(\mathrm{II})]$ and donor (bimane) was calculated using the Förster equation

$$
R=R_{0}\left(\frac{1}{E}-1\right)^{1 / 6}
$$

where $\mathrm{R}_{0}$ for bimane to $\mathrm{Cu}(\mathrm{II})$ is $12 \AA$ [15] [16] [19] [23]. 


\section{Results}

\section{Design of tmFRET experiment to monitor the closed and open conformation of RF1}

We used tmFRET, which bypasses some of the problems associated with classical FRET to monitor conformational changes in RF1 [15-17]. In tmFRET, a colored transition metal ion complex is coordinated between two histidine residues, which act as an energy acceptor for small organic fluorophores (Fig. 1). Due to the very low extinction coefficients of metals, their $R_{0}$ values are very short. Therefore, this method is ideal to monitor small-scale distance changes within a protein $(5-20 \AA)$. To guide the optimal location for the two histidine residues (His clamp) and the single cysteine to which the fluorophore will be attached, we used an automated homology-modeling program (ESyPred3D) to create a structural homolog of E. coli RF1 in a closed conformation using the crystal structure of Thermotoga maritima RF1 as the template (1RQ0) [3] [24]. Similarly, we created an E. coli structural homolog of the open conformation using the crystal structure of Thermus thermophilus RF1 bound to the ribosome as the template (3D5A) [8].

The structures of E. coli RF1 in the open and closed conformations were used to determine changes in the distance between every residue of RF1 in the both states (Fig. 2a-b). Briefly, the distance between the $\alpha$-carbon of each residue to all other residues were determined for the closed and open conformations of E. coli RF1 separately. In the next step, the change in distance between the closed and open conformations for each residue was plotted to create a two-dimensional distance map (Fig. 2c). This map shows the change in distance in the open and closed states for each and every residue pair in 
RF1. The color reflects the distance, ranging from $0 \AA$ to $50 \AA$. Residue pairs with no or only a small change (dark blue, $0 \AA$ to $5 \AA$ ) were classified as fixed pairs. These residues will not change their distance relative to each other when RF1 undergoes the closed to open conformational change. Residue pairs with a moderate or large change (light blue to yellow, $5 \AA$ to $50 \AA$ ) were considered dynamic pairs. The distances between the corresponding residues are different in the closed versus the open RF1 structures. Since tmFRET only gives reliable measurements between $5-20 \AA$, only pairs with distance changes close to this range were considered for mutagenesis.

To further select the best residues to place the His clamp, we performed a conservation analysis of $E$. coli RF1 to identify residues that are poorly conserved using the program ConSurf Server [25] [26] [27]. A structural model was created with colorcoded residues reflecting the level of conservation (Fig. 2d). Residues with low conservation that are on the surface of RF1 were considered suitable to introduce the mutations. Finally, a comparison of the closed and open RF1 structures showed that the long $\alpha$-helix in domain III collapses on top of domain II in the closed conformation, bringing the GGQ motif within $\sim 25 \AA$ of the PxT motif $[3,8]$, whereas, in the open conformation the $\alpha$-helix in domain III stretches out and away from domain II, placing the GGQ and PxT motifs $~ 75 \AA$ apart. Thus, the largest change in distance from a closed to an open state occurs between domains II and III of RF1.

We used our distance map and the conservation analysis to identify positions in domains II and III of RF1 that were suitable to introduce mutations for tmFRET pairs. An additional constraint of engineering the metal-binding His clamp lies in the fact that that the two histidine residues have to be located on an $\alpha$-helix separated by four amino 
acids ( $i$ and $i+4)$, which will place their imidazole groups next to each other to coordinate a metal ion bond. We selected A268 and S272 to be changed to histidines because they are poorly conserved and located on an $\alpha$-helix in domain III (Fig. 2d). We chose S167 to be changed to cysteine because it is not conserved and is on the surface of domain II. The distance between the His clamp (A268H, S272H) and S167C are $~ 15 \AA$ and $\sim 36 \AA$ in the closed and open conformations of RF1, respectively (Fig. 2e-f). The distance between the His clamp and $\mathrm{C} 167$ in the closed conformation is within the measurement range of tmFRET. However, in the open conformation the distance between these two positions is outside the measurable range for tmFRET. Nevertheless, we selected these positions because it was clear from the structural data that RF1 is in an open conformation when bound to the ribosome, but it is unclear whether RF1 is in the closed conformation without the ribosome. Therefore, we wanted a precise distance measurement for the closed conformation of RF1 at the expense of some uncertainty in distance measurement in the open conformation. We could not identify other suitable residues that were within the range for tmFRET in the closed and open conformations that met all of our above-mentioned requirements. We used a cysteine-free version of RF1 to introduce a single cysteine at position S167 and the pair of histidines at positions A268 and S272 by site-directed mutagenesis. We call this mutant protein the RF1 dynamic pair mutant (S167C/A268H/S272H).

To create an RF1 fixed pair mutant as a control for tmFRET experiments, we selected E256 for a change to cysteine because of its poor conservation, surface location and the distance to the His clamp (A268H, S272H), which is estimated to be $\sim 11-12 \AA$ in both conformations (Fig. 2g-h). We call this mutant protein the RF1 fixed pair mutant 
(E256C/A268H/S272H). Additionally, we made RF1 mutants S167C and E256C without a His clamp to be used as controls to account for solution quenching (that is, quenching without the coordinated metal ion).

\section{RF1 mutants are active in termination}

The mutant RF1 proteins were purified, and the fluorescent dye monobromobimane $(\mathrm{mBBr})$ was covalently linked to the single cysteine at $\mathrm{S} 167 \mathrm{C}$ or E256C. To test whether the bimane-labeled RF1 mutants are active, we carried out two functional assays. First, we analyzed the activity of RF1 mutants using a fluorescencebased ribosome-binding assay [22]. In this assay, the binding of RF1 to ribosomes programmed with a pyrene-labeled mRNA having a stop codon in the A site causes an increase in the fluorescence emission intensity of pyrene. Wild type RF1 and bimanelabeled RF1 mutants showed overall a similar increase in pyrene fluorescence intensity indicating that the mutations and the attachment of the fluorescent dye did not reduce RF1 binding activity (Fig. 3a). Second, we determined the rate of peptide release by wild type RF1 and RF1 mutants (Fig. 3b and c). Time course experiments for peptide release at different concentrations of $\mathrm{Cu}(\mathrm{II})(0$ to $100 \mu \mathrm{M})$ were performed with wild type RF1 and a bimane-labeled RF1 dynamic pair mutant (S167C/A268H/S272H). Wild type RF1 gave a rate of $0.15 \pm 0.01 \mathrm{~s}^{-1}$ in the absence of $\mathrm{Cu}$ (II) and a rate of $0.12 \pm 0.03 \mathrm{~s}^{-1}$ in the presence of $50 \mu \mathrm{M} \quad \mathrm{Cu}(\mathrm{II})$. The bimane-labeled $\mathrm{RF} 1$ dynamic pair mutant (S167C/A268H/S272H) gave a rate of $0.13 \pm 0.01 \mathrm{~s}^{-1}$ in the absence of $\mathrm{Cu}(\mathrm{II})$ and a rate of $0.12 \pm 0.03 \mathrm{~s}^{-1}$ in the presence of $50 \mu \mathrm{M} \mathrm{Cu}$ (II) (Fig. 3b). The peptide release rates did not decrease significantly with increasing concentrations of $\mathrm{Cu}$ (II) up to $100 \mu \mathrm{M}$ (Fig. 
3c).

\section{Conformational analysis of RF1 by tmFRET}

To verify that the design of our RF1 mutants is suitable for the tmFRET experiments we first analyzed the quenching of bimane by $\mathrm{Cu}(\mathrm{II})$ in the absence of the ribosome. An increasing amount of $\mathrm{Cu}(\mathrm{II})(0-10 \mathrm{mM})$ was added to a fixed concentration of bimane-labeled RF1 mutants. Samples were excited at $400 \mathrm{~nm}$, and the fluorescence intensity at $460 \mathrm{~nm}$ was measured. The obtained values were normalized to the initial value without $\mathrm{Cu}(\mathrm{II})$ and plotted as a function of $\mathrm{Cu}(\mathrm{II})$ concentration (Fig. 3d-f). $\mathrm{Cu}$ (II) quenching traces of the dynamic and the fixed pairs were compared to the corresponding cysteine-only control (Fig. 3e-f, solid and dashed trace, respectively). Quenching traces from cysteine-only controls could be fit to a single-site binding equation, where the observed quenching derives from collisional quenching by $\mathrm{Cu}(\mathrm{II})$ in solution. $\mathrm{RF} 1$ mutants with the His clamp showed a second phase of quenching at lower concentrations of $\mathrm{Cu}(\mathrm{II})$ and the corresponding quenching traces could be fit to a two-site binding model. This earlier quenching phase could only be observed with RF1 mutants with a His clamp and is therefore considered a result of tmFRET. These results validate that the design of the RF1 mutants is appropriate for monitoring the conformation of RF1 by tmFRET.

\section{Binding to the ribosome induces a closed to open conformational change in RF1}

We next compared the quenching behavior of the bimane-labeled RF1 mutants in the presence and absence of the release complex (Fig. 4). A fixed concentration of RF1 
mutants $(100 \mathrm{nM})$ were incubated with or without a fixed concentration of release complex (RC) (300 nM), which were programmed with either a UAA stop codon or a UUU sense codon in the ribosomal A site (Fig. 4a-e, red for UAA and blue for UUU). The fluorescence intensity at $460 \mathrm{~nm}$ was measured with increasing $\mathrm{Cu}(\mathrm{II})$ concentration. RF1 dynamic pair mutant (S167C/A268H/S272H) in the absence of RC showed an early, higher-affinity phase of quenching as a result of tmFRET (Fig. 4a, black curve). This is in agreement with what was observed above, and the data could be fit to a two-site binding model. When this RF1 mutant was incubated with RC that was programmed with a UAA stop codon in the A site, the early, higher-affinity quenching phase could not be observed any longer (Fig. 4a, red curve). Furthermore, the data could be fit to a onesite binding model suggesting that the His clamp is too far to quench the fluorescent dye significantly. In contrast, when the same RF1 mutant was incubated with RC that was programmed with a UUU sense codon in the ribosomal A site, the early, higher-affinity quenching phase was still observed (Fig. 4b, blue curve). The data could be fit to a twosite binding model and was comparable to the data obtained when this RF1 mutant was incubated without RC. This result suggests that RF1 was unable to bind to ribosomes with a UUU sense codon and is still in the original (closed) conformation. Comparing the quenching curves of RF1 dynamic pair mutant in the presence of RC with the UAA and UUU codons directly highlight the distinct quenching patterns (Fig. 4c). Importantly, we observed the largest difference in quenching efficiency at $50 \mu \mathrm{M} \mathrm{Cu}(\mathrm{II})$ with the RF1 dynamic pair mutant.

When RF1 fixed pair mutant (E256C/A268H/S272H) was incubated under increasing $\mathrm{Cu}(\mathrm{II})$ concentrations in the absence or presence of $\mathrm{RC}$ programmed with a 
UAA stop codon, no significant difference in quenching behavior could be detected (Fig. 4d). The data could be fit to a two-site binding model. This result suggests that the distance between the His clamp and the bimane-labeled single-cysteine is approximately the same in either conformation. This is consistent with data from the dynamic distance map where this pair is predicted not to change in distance in the closed and open conformation. Finally, the cysteine-only RF1 mutant (S167C) that was used as a control showed overall the same quenching behavior when incubated with or without RC and could be fit to a one-site binding model (Fig. 4e).

As mentioned above, the largest difference in quenching efficiency was observed at $50 \mu \mathrm{M} \mathrm{Cu}(\mathrm{II})$, which was used to determine the FRET efficiency and the distance between the His clamp and the bimane fluorophore (R-value) for the RF1 mutants. In the absence of RC for the RF1 dynamic pair mutant (Fig. 4a), the FRET efficiency was 0.306 and the R-value was $13.7 \AA$, which closely matches the distance measured from the RF1 structure in the closed conformation (15 A, Fig. 2e). For RF1 fixed pair mutant (Fig. 4d), the FRET efficiency was 0.377 and the R-value was $13 \AA$, which also precisely matches the distance measured from the RF1 structure in the closed conformations (12 $\AA$, Fig. 2g). However, in the presence of saturating concentration of RC programmed with a UAA codon (Fig. 4a), the FRET efficiency (E) for the RF1 dynamic pair mutant was 0.043 , which corresponds to an R-value of $20.2 \AA$. The R-value is lower than the expected distance in the open state ( $36 \AA$, Fig. $2 \mathrm{f}$ ) because tmFRET is not very sensitive beyond $\approx 20 \AA$ as discussed above. When the RF1 dynamic pair mutant was incubated under an identical condition with UUU programmed RC (Fig. 4b), the FRET efficiency (E) was 0.334 , which corresponds to an R-value of $13 \AA$. This distance measurement is 
in good agreement with the expected distance of $15 \AA$ in the closed state of RF1. This result confirms that RF1 was unable to bind to the ribosome under these conditions, and the histidine clamp remains close enough to the fluorescent dye to result in significant quenching. The RF1 fixed pair mutant showed a FRET efficiency of 0.201 in the presence of RC programmed with a UAA codon (Fig. 4d). This corresponds to an Rvalue of $15.1 \AA$, which is slightly larger than the calculated distance of $11 \AA$ from the RF1 structure in the open conformation (Fig. 2h).

To investigate the conformational change as a function of $\mathrm{RC}$ concentration, a fixed concentration of mutant RF1 (100 nM) were incubated with a fixed concentration of $\mathrm{Cu}$ (II) $(50 \mu \mathrm{M})$ and an increasing concentration of $\mathrm{RC}(0-400 \mathrm{nM})$. RCs were again either programmed with a UAA stop codon or a UUU sense codon in the ribosomal A site (Fig. 4f). In all cases, we observed an increase in fluorescence intensity with increasing concentrations of ribosomes due to background from light scattering. These background values were subtracted from all data sets. As a control, the cysteine-only RF1 mutant (S167C, Fig. 4f, circles) was used where no signal change was expected upon binding to the ribosome. Nevertheless, we could observe a 2-fold increase in fluorescence intensity with increasing amounts of $70 \mathrm{~S}$ ribosomes programmed with a UAA codon in the A site. This increase in fluorescence may be due to changes in the environment of the bimane fluorophore when RF1 binds to the ribosome.

A similar change in fluorescence intensity could be observed with the RF1 fixed pair mutant (Fig. 4f, diamonds). This result reinforces the hypothesis that the 2 -fold increase in fluorescence is due to changes in the environment of the fluorophore as a result of RF1 binding to the ribosome and is not due to a change in tmFRET. 
Interestingly, the RF1 dynamic pair mutant showed a 3.5 -fold increase in fluorescence intensity with increasing concentrations of RC programmed with UAA (Fig. 4f, triangle). We attribute this distinct effect over the other tested RF1 mutants to an increase in the distance between the coordinated metal ion and the fluorescent dye upon RF1 binding to the ribosome. The predicted closed to open conformational change will position the coordinated $\mathrm{Cu}$ (II) ion further away from the bimane dye, thereby diminishing the quenching effect observed in the absence of ribosomes. When we incubated the same RF1 mutant with increasing amounts of RC programmed with a UUU codon in the A site (Fig. 4f, squares), we only observed a 1.5 -fold increase in fluorescence intensity. The net reduced fluorescence intensity is qualitatively consistent with a large $K_{D}$ difference for RF1 binding to UUU codon compared to UAA codon in the A site, and RF1 is not expected to bind to RC under these conditions and, therefore, remains in the closed conformation [22]. 


\section{Discussion}

In all organisms, termination of protein synthesis starts with the recognition of a stop codon in the ribosomal A site by class I release factors [1]. Stop codon recognition has to be precise because premature termination on sense codons will lead to the synthesis of non-functional truncated proteins, which could be toxic to the cell. The accuracy of stop codon recognition by RF1 and RF2 are $\approx 10^{-5}$ and is similar to the accuracy of tRNA selection by the ribosome [28-30]. However, tRNA selection uses proofreading to attain high fidelity, whereas RF1 and RF2 do not use proofreading and the molecular basis for avoiding erroneous termination events with sense codons in the ribosomal A site is not clear [29, 31, 32]. It was previously proposed that RF1 and RF2 in the closed conformation bind with low affinity to the ribosome and dissociates with high probability if they encounter a sense codon in the A site [5]. In contrast, if RF1 and RF2 encounter a stop codon in the A site, they switch to the open conformation, which has high binding affinity for the ribosome and properly orients the GGQ motif in the peptidyl transferase center to trigger the release of the newly synthesized protein attached to the peptidyl-tRNA [5]. Thus, according to this hypothesis, an induced fit mechanism is used by RF1 and RF2 to discriminate sense and stop codons. However, this hypothesis contradicts SAXS data indicating that $>90 \%$ of $E$. coli RF1 in solution is in the open conformation [13].

Surprisingly, another SAXS analysis of T. thermophilus RF2 indicates that the protein free in solution is a mixture of $80 \%$ closed and $20 \%$ open conformation [14]. Additionally, crystal structures of RF1 and RF2 from E. coli and other species obtained 
under a variety of crystallization conditions are all in the closed conformation arguing that the closed conformation is not an artifact but is of functional relevance $[3,4,14]$. Indeed, RF2 bound to the methyltransferase PrmC has the closed conformation [12]. Moreover, the open conformation is thermodynamically unstable unless bound to the ribosome, because it exposes conserved hydrophobic residues at the interface between domains III and II/IV [5]. Collectively, these data suggest that RF1 and RF2 favor a closed conformation in the absence of the ribosome.

To monitor the conformation of RF1 in the absence and presence of the ribosome with stop or sense codons in the A site, we used tmFRET. Our data clearly show that RF1 free in solution is mainly in the closed conformation and the equilibrium is shifted to the open conformation in the presence of ribosomes with a stop codon in the A site. More importantly, tmFRET analysis with the RF1 dynamic pair mutant in the absence of the ribosome showed that the distance between the His clamp and the bimane dye is 13.7 $\AA$. This result is in excellent agreement with the $15 \AA$ distance measured in the closed RF1 structure. Furthermore, a clear increase in distance between the two sites was observed in the presence of the ribosome with a stop codon in the A site indicating a change from a compact to a more extended RF1 structure. These results show that $E$. coli RF1 is mainly in the closed conformation in solution.

Our tmFRET data contradicts the SAXS data for E. coli RF1 [13]. One possibility is that domains I and III of RF1 are very dynamic relative to static domains II/IV making it difficult to interpret the SAXS data [3]. An attempt was made to overcome this problem by deleting domain I from RF1 and using the truncated RF1 for interpreting the SAXS data of the wild type RF1. However, it is difficult to rule out the 
possibility that deleting domain I stabilize the open conformation of RF1 in solution and it may not be appropriate to use truncated RF1 to interpret SAXS data of the wild type RF1. SAXS analysis determines the global structure of the protein and is strongly influenced by structural changes anywhere in the protein. In contrast, tmFRET provides the exact distance between two positions in the protein and is largely independent of structural changes elsewhere in the protein [17].

Our results indicate that RF1 undergoes a conformational change upon binding to the ribosome with a stop codon but not with a sense codon in the A site. This is consistent with previous kinetic studies that showed that the dissociation rate constant ( $\left.k_{\text {off }}\right)$ of RF1 is as much as 4000 -fold greater with sense codons compared to a stop codon [22]. In other words, the large difference in the $k_{\text {off }}$ suggests that the structure of RF1 bound to the ribosome is different with a stop versus a sense codon in the A site. The distance that we measured with the RF1 dynamic pair mutant and RC with a UUU sense codon is $13 \AA$, which corresponds to the distance between these two positions in the closed state of RF1. Under these conditions, the distance does not change much because the $\mathrm{K}_{\mathrm{D}}$ for RF1 binding to RC with UUU codon is $1.6 \pm 0.3 \mu \mathrm{M}$ and there is little to no binding of RF1 to the ribosome at the concentrations used for the tmFRET experiment [22]. We could not perform the tmFRET experiments at higher concentrations of RC because the quantum efficiency of the bimane dye is low, and light scattering by ribosomes begin to dominate the fluorescence signal. Nonetheless, RC with the UUU sense codon serves as an excellent control for monitoring the conformation of RF1 bound to $\mathrm{RC}$ with a stop codon in the A site. 
As expected, the distance between the His clamp in domain II and the bimane dye in domain III of RF1 increases to $>20.2 \AA$ when it binds to the ribosome with a stop codon in the A site. We assume this value is not the precise distance between the His clamp and the bimane dye of the RF1 dynamic pair mutant because the expected distance change is $36 \AA$, which is greater than the upper limit of the tmFRET method (5 to $20 \AA$ range) [17]. This is a limitation of our tmFRET study; however, it is not a serious limitation because there are several high-resolution structures of RF1 bound to the ribosome with a stop codon in the A site making it clear that RF1 is in the open conformation on the ribosome [8, 33].

Interestingly, directed hydroxyl radical probing data indicated that RF1 has different conformations on the ribosome depending on whether the A site has a stop or sense codon [34]. Importantly, the GGQ motif is mispositioned with respect to the peptidyl transferase center suggesting that authentic stop codon recognition is essential for correctly positioning RF1/RF2 for catalysis. Taken together, our tmFRET results and previous kinetic studies [22, 35], suggest that conformational changes by RF1 and RF2 are important for high fidelity stop codon recognition. We propose that RF1 and RF2 are mainly in the closed conformation in solution and interact initially with the ribosome in the closed conformation (Fig. 5). Interactions with the ribosome may induce RF1 and RF2 to adopt a partially open conformation to avoid a steric clash with ribosomal elements [8, 10]. However, RF1 and RF2 dissociate rapidly from the ribosome with sense codons in the A site because they fail to induce the final fully open conformation [34]. In contrast, the presence of a stop codon in the ribosomal A site will induce the open conformation of RF1 and RF2, which bind then with high affinity to the ribosome 
and positions the GGQ motif correctly for catalysis. Thus, conformational change by RF1 and RF2 that is coupled to stop codon recognition may be the molecular basis for high fidelity stop codon recognition during termination.

\author{
Acknowledgements \\ This work was supported by National Science Foundation grant 1158127 to S.J. and an \\ Erwin Schroedinger Fellowship from the Austrian Science Found to K.T. We thank \\ Ruben Gonzalez for the cysteine-free RF1 construct, Justin Taraska, Michael Puljung and \\ Michael Tauber for discussions on tmFRET analysis, Roy Wollman and $\mathrm{Ti} \mathrm{Wu}$ for \\ creating the dynamic distance map, and Uli Muller for comments on the manuscript.
}




\section{References}

[1] Capecchi MR. Polypeptide chain termination in vitro: isolation of a release factor. Proc Natl Acad Sci U S A. 1967;58:1144-51.

[2] Scolnick E, Tompkins R, Caskey T, Nirenberg M. Release factors differing in specificity for terminator codons. Proc Natl Acad Sci U S A. 1968;61:768-74.

[3] Shin DH, Brandsen J, Jancarik J, Yokota H, Kim R, Kim SH. Structural analyses of peptide release factor 1 from Thermotoga maritima reveal domain flexibility required for its interaction with the ribosome. J Mol Biol. 2004;341:227-39.

[4] Vestergaard B, Van LB, Andersen GR, Nyborg J, Buckingham RH, Kjeldgaard M. Bacterial polypeptide release factor RF2 is structurally distinct from eukaryotic eRF1. Mol Cell. 2001;8:1375-82.

[5] Rawat UB, Zavialov AV, Sengupta J, Valle M, Grassucci RA, Linde J, et al. A cryoelectron microscopic study of ribosome-bound termination factor RF2. Nature. 2003;421:87-90.

[6] Klaholz BP, Pape T, Zavialov AV, Myasnikov AG, Orlova EV, Vestergaard B, et al. Structure of the Escherichia coli ribosomal termination complex with release factor 2 . Nature. 2003;421:90-4.

[7] Korostelev A, Asahara H, Lancaster L, Laurberg M, Hirschi A, Zhu J, et al. Crystal structure of a translation termination complex formed with release factor RF2. Proc Natl Acad Sci U S A. 2008;105:19684-9.

[8] Laurberg M, Asahara H, Korostelev A, Zhu J, Trakhanov S, Noller HF. Structural basis for translation termination on the 70S ribosome. Nature. 2008;454:852-7.

[9] Jin H, Kelley AC, Loakes D, Ramakrishnan V. Structure of the 70S ribosome bound to release factor 2 and a substrate analog provides insights into catalysis of peptide release. Proc Natl Acad Sci U S A. 2010;107:8593-8.

[10] Weixlbaumer A, Jin H, Neubauer C, Voorhees RM, Petry S, Kelley AC, et al. Insights into translational termination from the structure of RF2 bound to the ribosome. Science. 2008;322:953-6.

[11] Ito K, Ebihara K, Uno M, Nakamura Y. Conserved motifs in prokaryotic and eukaryotic polypeptide release factors: tRNA-protein mimicry hypothesis. Proc Natl Acad Sci U S A. 1996;93:5443-8.

[12] Graille M, Heurgue-Hamard V, Champ S, Mora L, Scrima N, Ulryck N, et al. Molecular basis for bacterial class I release factor methylation by PrmC. Mol Cell. 2005;20:917-27.

[13] Vestergaard B, Sanyal S, Roessle M, Mora L, Buckingham RH, Kastrup JS, et al. The SAXS solution structure of RF1 differs from its crystal structure and is similar to its ribosome bound cryo-EM structure. Mol Cell. 2005;20:929-38. 
[14] Zoldak G, Redecke L, Svergun DI, Konarev PV, Voertler CS, Dobbek H, et al. Release factors 2 from Escherichia coli and Thermus thermophilus: structural, spectroscopic and microcalorimetric studies. Nucleic Acids Res. 2007;35:1343-53.

[15] Taraska JW, Puljung MC, Olivier NB, Flynn GE, Zagotta WN. Mapping the structure and conformational movements of proteins with transition metal ion FRET. Nat Methods. 2009;6:532-7.

[16] Taraska JW, Puljung MC, Zagotta WN. Short-distance probes for protein backbone structure based on energy transfer between bimane and transition metal ions. Proc Natl Acad Sci U S A. 2009;106:16227-32.

[17] Yu X, Wu X, Bermejo GA, Brooks BR, Taraska JW. Accurate high-throughput structure mapping and prediction with transition metal ion FRET. Structure. 2013;21:919.

[18] Puljung MC, Zagotta WN. A secondary structural transition in the C-helix promotes gating of cyclic nucleotide-regulated ion channels. J Biol Chem. 2013;288:12944-56.

[19] Selvin PR. Fluorescence resonance energy transfer. Methods Enzymol. 1995;246:300-34.

[20] Powers T, Noller HF. A functional pseudoknot in 16S ribosomal RNA. EMBO J. 1991;10:2203-14.

[21] Studer SM, Joseph S. Binding of mRNA to the bacterial translation initiation complex. Methods Enzymol. 2007;430:31-44.

[22] Hetrick B, Lee K, Joseph S. Kinetics of stop codon recognition by release factor 1 . Biochemistry. 2009;48:11178-84.

[23] Taraska JW, Zagotta WN. Structural dynamics in the gating ring of cyclic nucleotide-gated ion channels. Nat Struct Mol Biol. 2007;14:854-60.

[24] Lambert C, Leonard N, De Bolle X, Depiereux E. ESyPred3D: Prediction of proteins 3D structures. Bioinformatics. 2002;18:1250-6.

[25] Ashkenazy H, Erez E, Martz E, Pupko T, Ben-Tal N. ConSurf 2010: calculating evolutionary conservation in sequence and structure of proteins and nucleic acids. Nucleic Acids Res. 2010;38:W529-33.

[26] Landau M, Mayrose I, Rosenberg Y, Glaser F, Martz E, Pupko T, et al. ConSurf 2005: the projection of evolutionary conservation scores of residues on protein structures. Nucleic Acids Res. 2005;33:W299-302.

[27] Glaser F, Pupko T, Paz I, Bell RE, Bechor-Shental D, Martz E, et al. ConSurf: identification of functional regions in proteins by surface-mapping of phylogenetic information. Bioinformatics. 2003;19:163-4.

[28] Jorgensen F, Adamski FM, Tate WP, Kurland CG. Release factor-dependent false stops are infrequent in Escherichia coli. J Mol Biol. 1993;230:41-50.

[29] Freistroffer DV, Kwiatkowski M, Buckingham RH, Ehrenberg M. The accuracy of codon recognition by polypeptide release factors. Proc Natl Acad Sci U S A. 2000;97:2046-51. 
[30] Bouadloun F, Donner D, Kurland CG. Codon-specific missense errors in vivo. EMBO J. 1983;2:1351-6.

[31] Ninio J. Kinetic amplification of enzyme discrimination. Biochimie. 1975;57:58795.

[32] Pape T, Wintermeyer W, Rodnina MV. Complete kinetic mechanism of elongation factor Tu-dependent binding of aminoacyl-tRNA to the A site of the E. coli ribosome. EMBO J. 1998;17:7490-7.

[33] Korostelev AA. Structural aspects of translation termination on the ribosome. RNA. 2011;17:1409-21.

[34] He SL, Green R. Visualization of codon-dependent conformational rearrangements during translation termination. Nat Struct Mol Biol. 2010;17:465-70.

[35] Field A, Hetrick B, Mathew M, Joseph S. Histidine 197 in release factor 1 is essential for a site binding and peptide release. Biochemistry. 2010;49:9385-90. 


\section{Figure Legends}

Figure 1. Illustration of tmFRET method. To monitor conformational change in RF1 (red), a bimane fluorescent dye (yellow star) is attached to a single cysteine and a $\mathrm{Cu}$ (II) ion (gray sphere) is coordinated by two histidine residues (His clamp, black sticks). In the $\mathrm{RF} 1$ closed conformation, the $\mathrm{Cu}$ (II) ion is close to the bimane dye resulting in low fluorescence because of quenching (left side). In the RF1 open conformation, the $\mathrm{Cu}$ (II) ion moved away from the bimane dye resulting in less quenching and increased fluorescence (right side).

Figure 2. Mapping the change in RF1 structure. (a) Crystal structure of Thermotoga maritima RF1 in a closed conformation showing the GGQ motif (yellow spheres) that is important for catalysis and the PXT motif (green spheres) that is important for stop codon recognition (PDB code: 1RQ0). The four domains in RF1 are indicated in different colors as follows: I (blue and cyan), II (green), III (orange and yellow) and IV (red). (b) Crystal structure of Thermus thermophilus RF1 in an open conformation (PDB code: 3D5A). (c) Distance map comparing the open and closed conformation of E. coli RF1 homology models. The color code shows the amino acid to the amino acid difference in distance between both states of RF1. The circled positions show the pairs of residues used to create the RF1 dynamic (S167C/A268H/S272H) and fixed (E256C/A268H/S272H) pair mutants for tmFRET studies. For comparison, the $\mathrm{GGQ} / \mathrm{PXT}$ change in distance is also indicated. (d) Conservation analysis of E. coli RF1. Colors of residues reflect the level of conservation $(0-9)$. The residues that were mutated for tmFRET study are shown as spheres and for comparison the GGQ motif and 
PXT motif are shown. (e and f) The distance between single cysteine (S167C) and the His clamp (H268/H272) in the closed and open conformations are indicated for the RF1 dynamic pair mutant (dashed line, $15 \AA$ and $36 \AA$, respectively). (g and h) Distance between single cysteine (E256C) and the His clamp (H268/H272) in the closed and open

conformation are indicated for the RF1 fixed pair mutant (dashed line, $12 \AA$ and $11 \AA$, respectively). Single cysteine is shown as blue spheres, and the His clamp is shown as red spheres.

\section{Figure 3. RF1 activity assays and tmFRET measurements of RF1 in the absence of}

ribosome. (a) RF1 mutants are active in binding to ribosomes. Binding of wild type RF1 and bimane-labeled RF1 mutants to Release Complex (RC) was monitored by the increase in the fluorescence intensity of pyrene-labeled mRNA. RC programmed with a UAA codon in the ribosomal A site were incubated without (dotted line) and with wild type RF1 (red line) and RF1 mutants (blue line is the dynamic pair, and the green line is the fixed pair). Samples were excited at $343 \mathrm{~nm}$, and the increase in the emission peak at $376 \mathrm{~nm}$ was used to monitor RF1 binding to RC (indicated by arrow). (b) Representative examples of peptide release time courses. Wild type RF1 in the absence of $\mathrm{Cu}$ (II) (open circles) and with $50 \mu \mathrm{M} \mathrm{Cu}$ (II) (filled circles). Bimane-labeled RF1 dynamic pair mutant in the absence of $\mathrm{Cu}$ (II) (open triangle) and with $50 \mu \mathrm{M} \mathrm{Cu}$ (II) (filled triangle). Data were normalized and fit to single exponential equation. (c) The rate of peptide release for wild type RF1 (red line) and the bimane-labeled RF1 dynamic pair mutant (blue line) under increasing amounts of $\mathrm{Cu}$ (II) $(0-100 \mu \mathrm{M})$. (d) A schematic illustration showing the expected quenching curve with increasing amounts of $\mathrm{Cu}(\mathrm{II})$. Idealized quenching 
curve for the cysteine-only protein (dashed line) and protein with cysteine and His clamp (solid line). The quenching curve can be used to determine the FRET efficiency and the $K_{\mathrm{d}}$ for $\mathrm{Cu}(\mathrm{II})$ binding to the histidine clamp. Y-axis is the normalized fluorescence signal in the presence of $\mathrm{Cu}$ (II) divided by signal without $\mathrm{Cu}$ (II). (e) Quenching curves of RF1 dynamic pair mutant (solid line) and the corresponding cysteine-only control (dashed line). Error bars represent standard deviation from at least 6 individual traces. (f) Quenching curves of RF1 fixed pair mutant (solid line) and the corresponding cysteine-only control (dashed line). Error bars represent standard deviation from at least 6 individual traces.

Figure 4. tmFRET measurements of RF1 in the presence of ribosome. (a) Quenching curves of RF1 dynamic pair mutant with RC having UAA stop codon in the A site (red line, solid triangles) and without RC (black line, open triangles). (b) Quenching curves of RF1 dynamic pair mutant with RC having UUU sense codon in the A site (blue line, solid squares) and without RC (black line, open triangles). (c) Overlay of RF1 dynamic pair mutant with RC having UAA stop codon in the A site (red line, solid triangles) and with RC having UUU sense codon in the A site (blue line, solid squares). (d) Quenching curves of RF1 fixed pair mutant with RC having UAA stop codon in the A site (red line, solid diamonds) and without RC (black line, open diamonds). (e) Quenching curves of cysteine-only RF1 (C167) with RC having UAA stop codon in the A site (red line, solid circles) and without RC (black line, open circles). (f) Conformational change in RF1 induced by RC titration. A fixed concentration of the RF1 dynamic pair mutant was incubated with increasing amounts of RC having UAA stop codon (red line, triangles) or 
UUU sense codon (blue line, squares) in the A site. A fixed concentration of the cysteine-only RF1 (C167) (red line, circles) and the RF1 fixed pair mutant (red line, diamonds) were incubated with increasing amounts of RC having UAA stop codon. The concentration of $\mathrm{Cu}(\mathrm{II})$ was $50 \mu \mathrm{M}$ in these experiments. Fluorescence signal was transformed to initial values without $\mathrm{Cu}$ (II). Error bars represent standard deviation from at least 3 individual experiments.

\section{Figure 5. Model for stop codon recognition and conformational change by RF1.}

RF1 is in the closed conformation in solution. Binding to the ribosome with a stop codon in the A site induces the open conformation of RF1 and correctly positions the GGQ motif in the 50S ribosomal subunit for catalysis. In contrast, binding to the ribosome with a sense codon in the A site fail to stabilize the open conformation of RF1 and RF1 dissociates rapidly from the ribosome. RF1 is in red, 50S subunit is in gray, 30S subunit is in cyan, mRNA is in dark blue, peptidyl-tRNA is in green and the newly synthesized protein attached to it is shown as blue circles. 
Closed

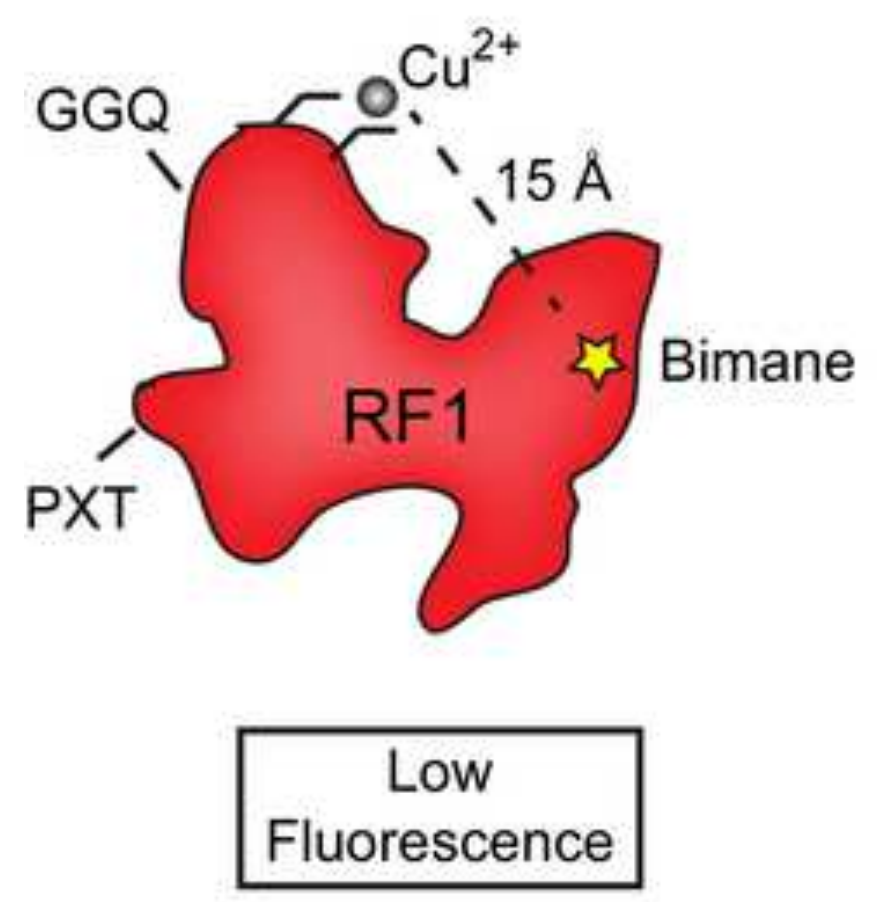

Open

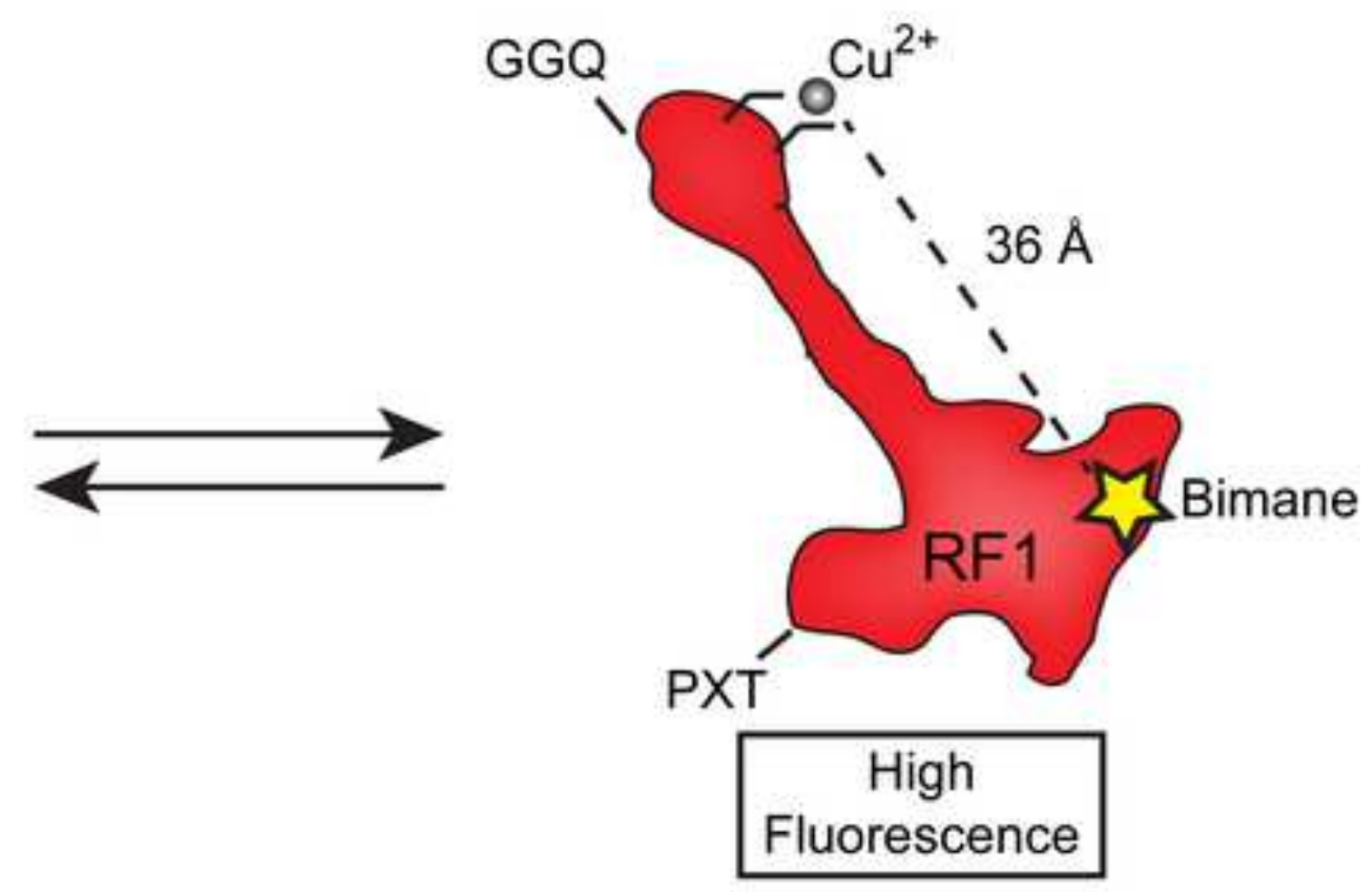

Figure 1 
(a)

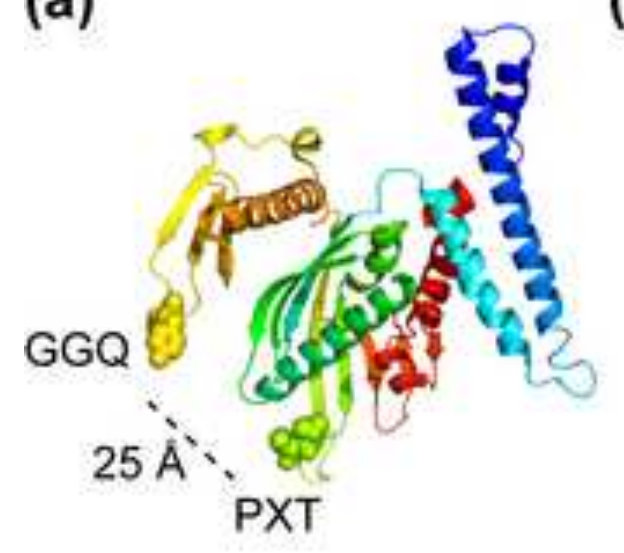

(b)

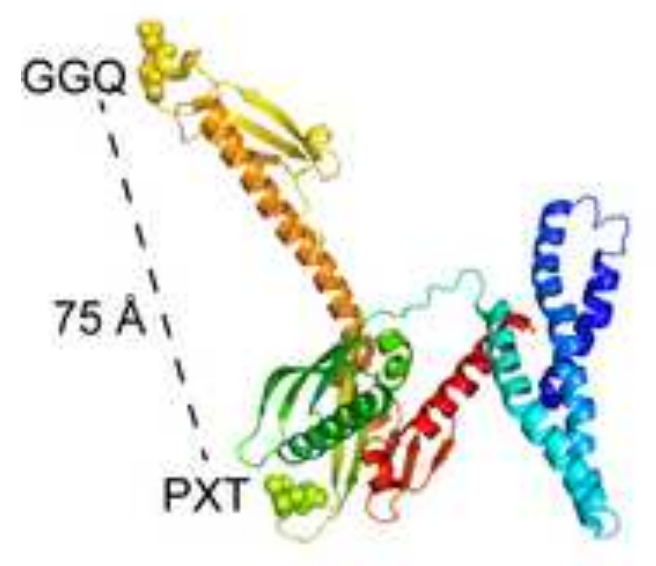

(d)

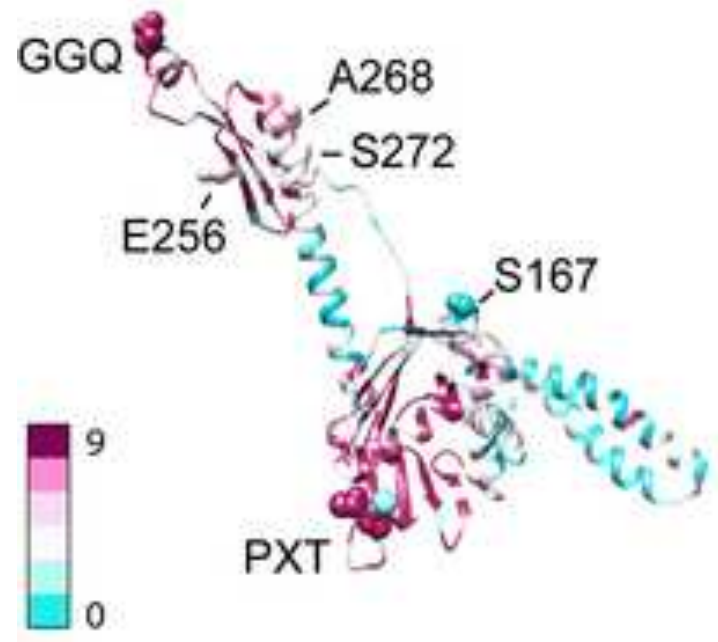

(c)

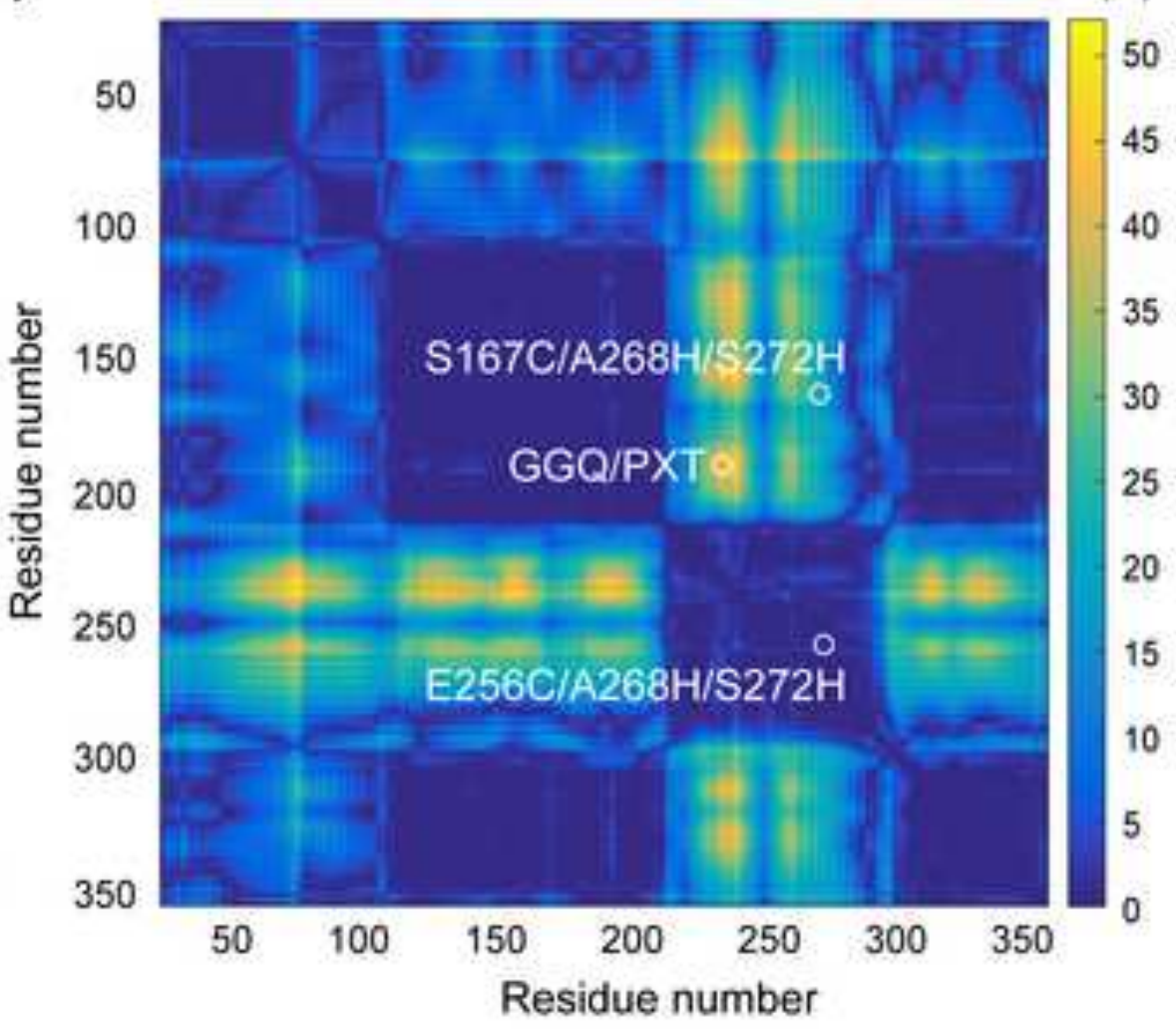

(e)

(f)

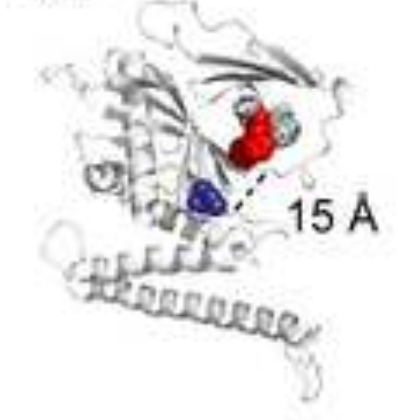

(g)

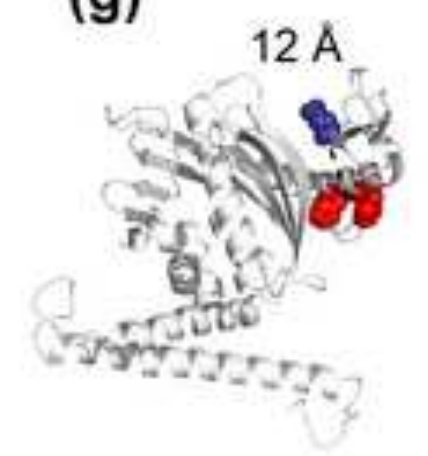

(h)

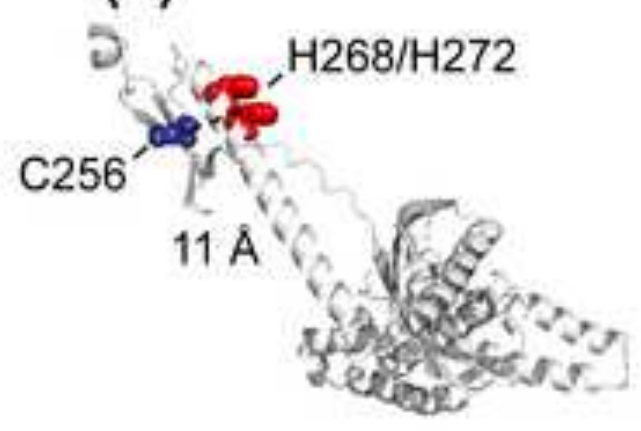

Figure 2 
(a)

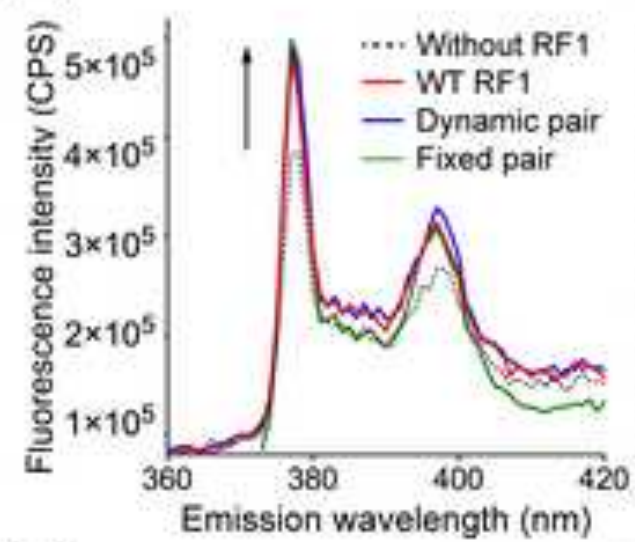

(d)

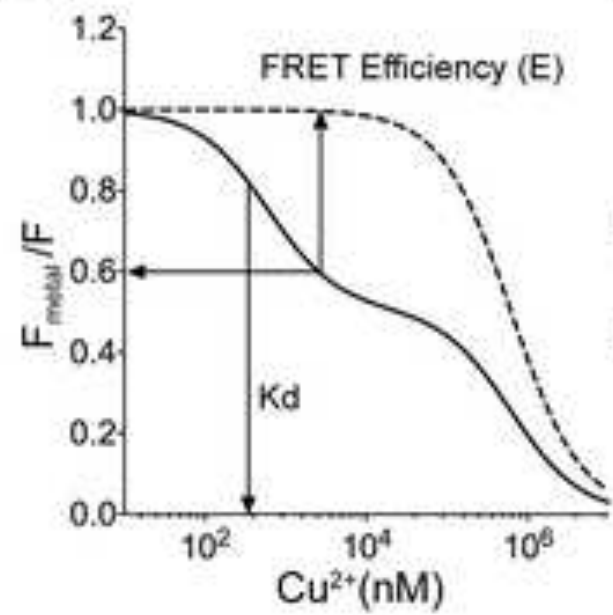

(b)

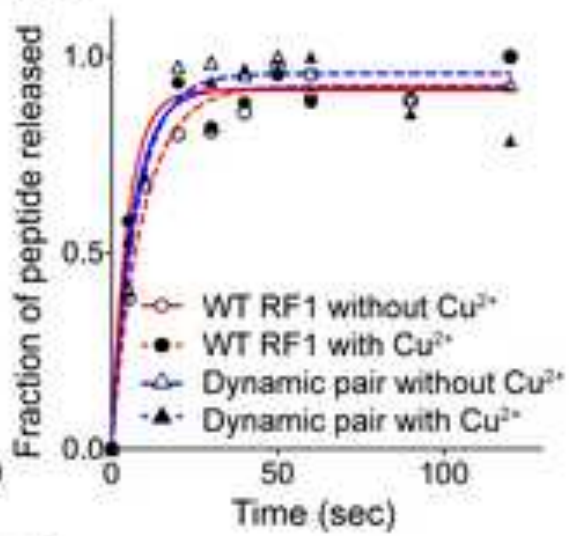

(e)

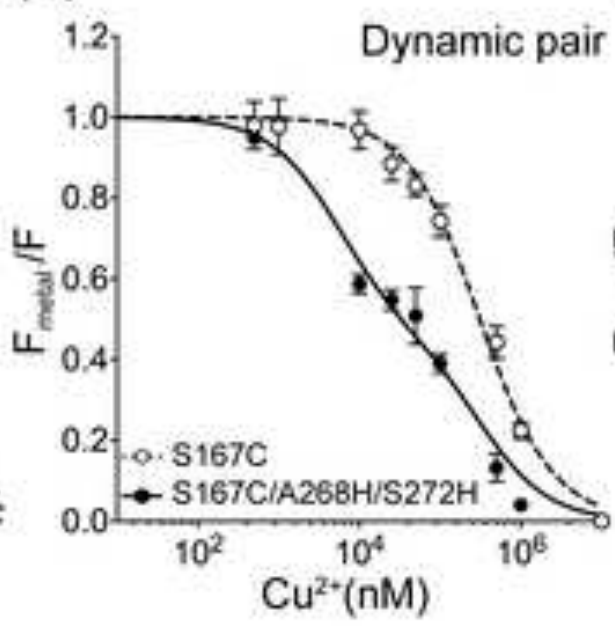

(c)

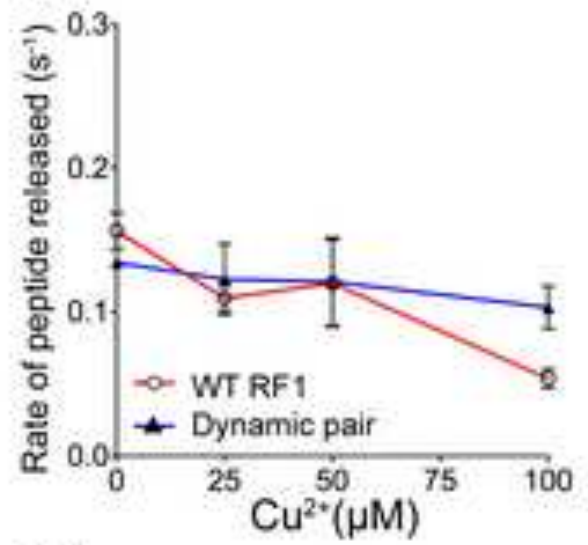

(f)

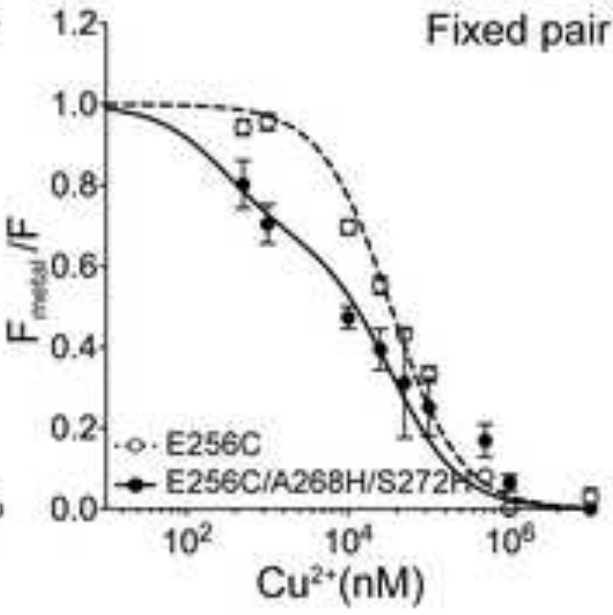

Figure 3 
(a)

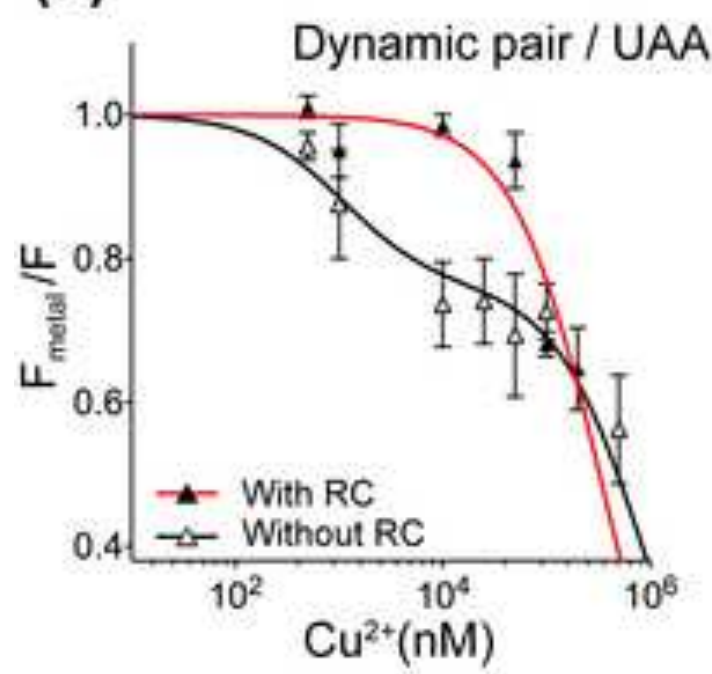

(d)

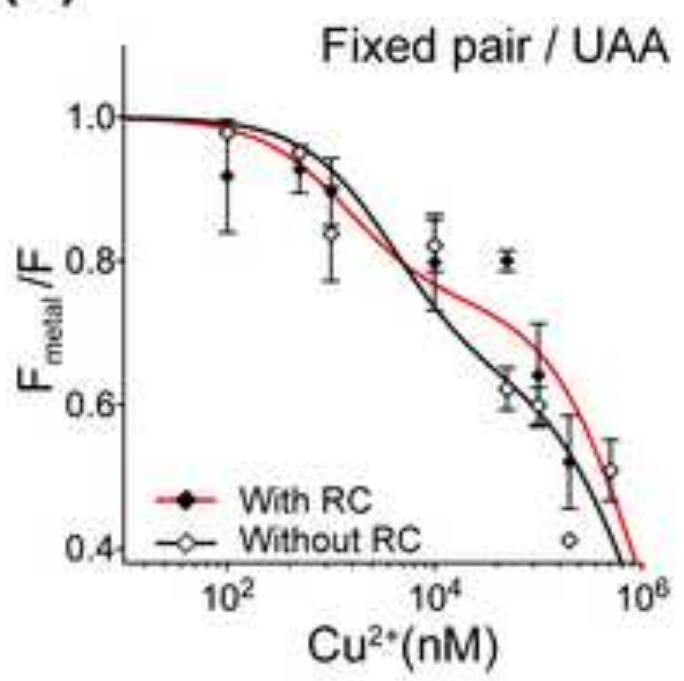

(b)

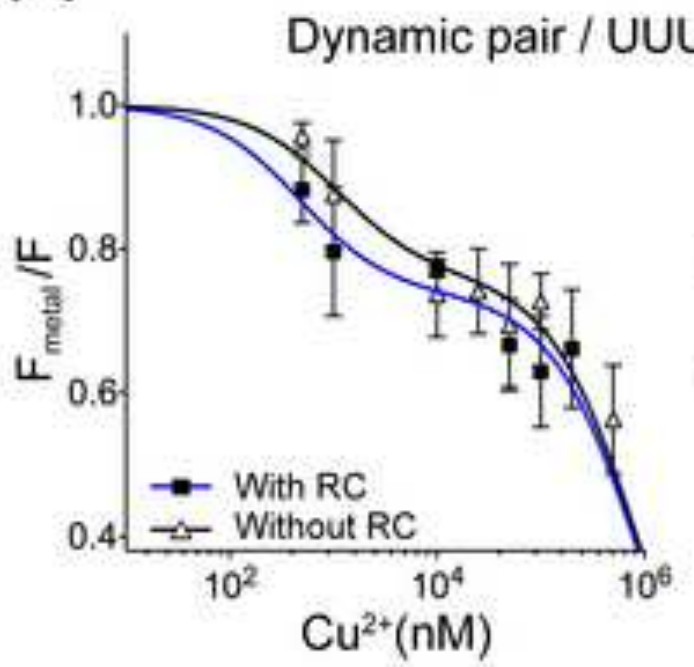

(e)

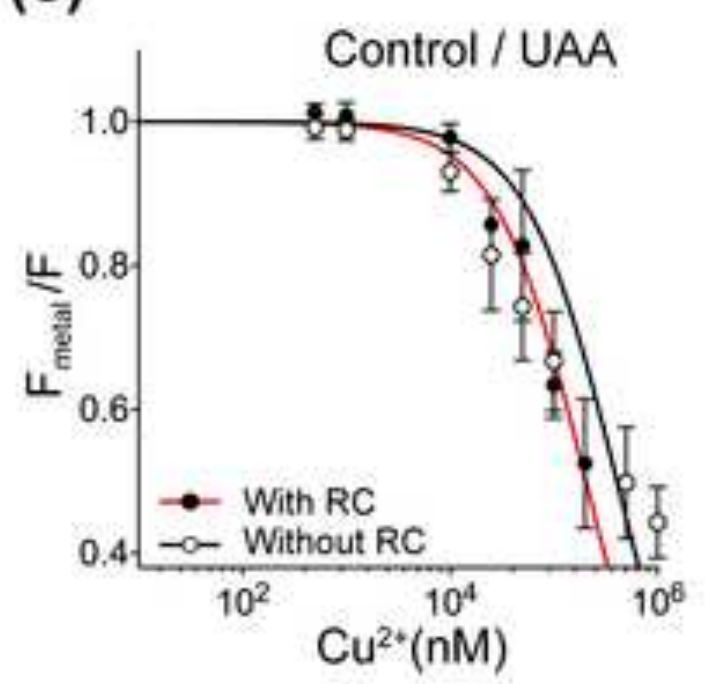

(c)

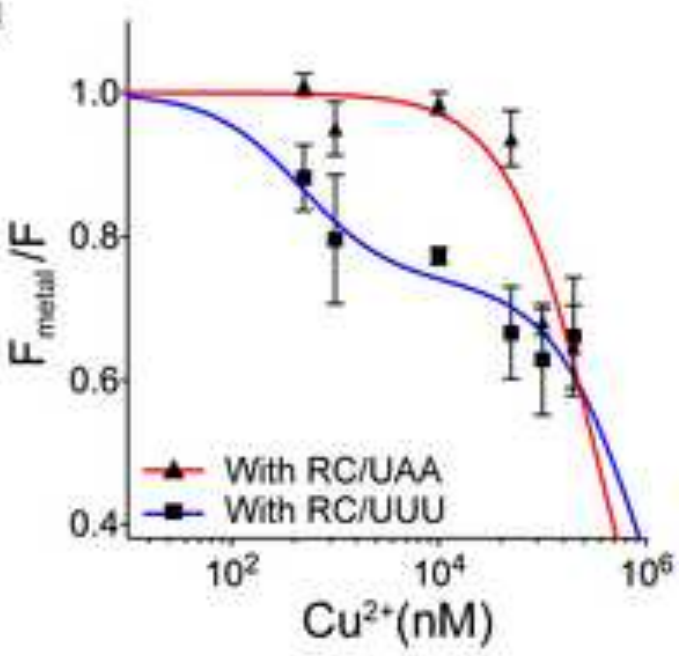

(f)

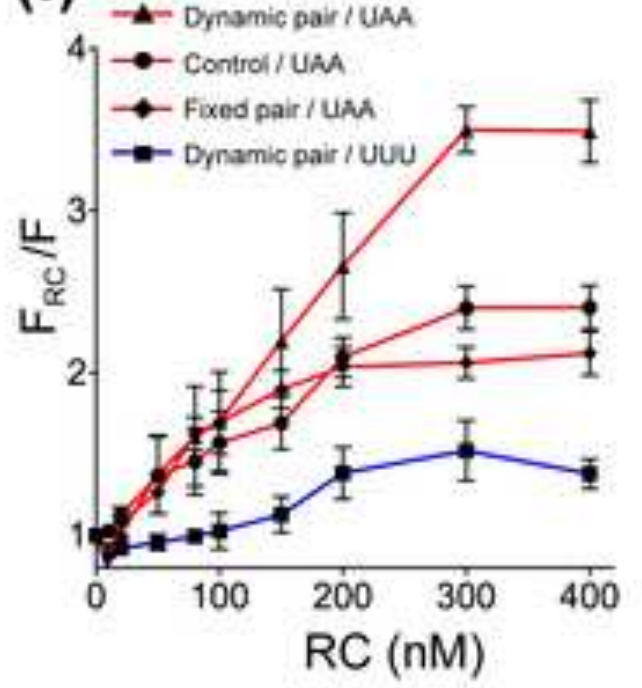

Figure 4 


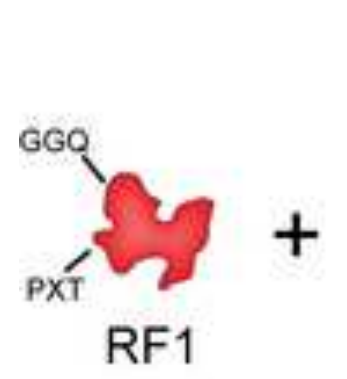

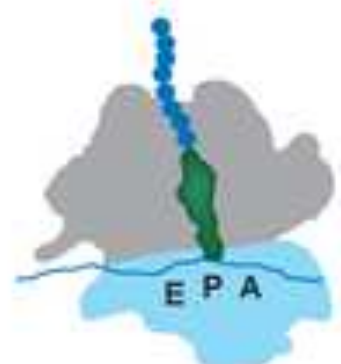

RC
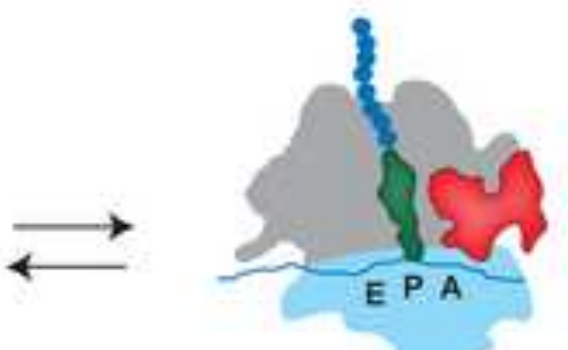

Initial

binding

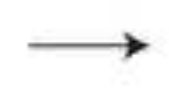

Stop codon recognition \& conformational change

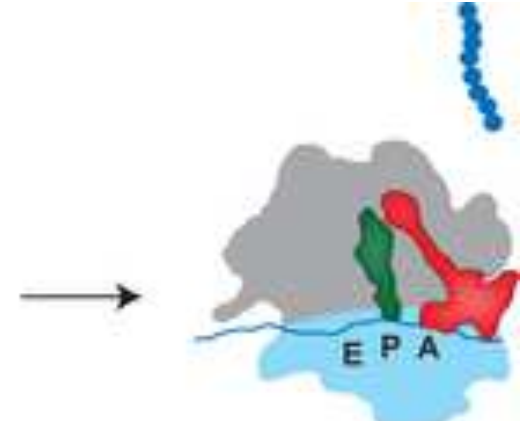

Peptide release

Figure 5 